\title{
Validating scores predicting atrial fibrillation recurrence post catheter ablation in patients with concurrent atrial fibrillation and pulmonary diseases
}

\author{
Ying Bai ${ }^{1}$, Zhen-Zhou Wang ${ }^{2}$, Gai-Gai Zhang ${ }^{3}$, Shi-Dong Guo ${ }^{4}$, José Miguel Rivera-Caravaca ${ }^{5}$, \\ Yue-Li Wang ${ }^{6}$, Yuan-Yuan Jin ${ }^{7}$, Yue Liu ${ }^{7}$ \\ ${ }^{1}$ Cardiovascular Center, Beijing Tongren Hospital, Capital Medical University, Beijing, China; ${ }^{2}$ National Center for Trauma Medicine, Key \\ Laboratory of Trauma and Neural Regeneration, Trauma Center, Peking University People's Hospital, Beijing, China; ${ }^{3}$ Department of Geriatrics, \\ Beijing Huaxin Hospital, Tsinghua University, Beijing, China; ${ }^{4}$ Emergency Department of China-Japan Friendship Hospital, Beijing, China; \\ ${ }^{5}$ Department of Hematology and Clinical Oncology, Hospital General Universitario Morales Meseguer, Instituto Murciano de Investigación \\ Biosanitaria (IMIB-Arrixaca), Murcia, Spain; ${ }^{6}$ Department of Echocardiography, Beijing Anzhen Hospital, Capital Medical University, Beijing, \\ China; ${ }^{7}$ Department of Cardiology, The First Affiliated Hospital of Harbin Medical University, Harbin, China \\ Contributions: (I) Conception and design: Y Bai, Y Liu; (II) Administrative support: Y Bai, Y Liu; (III) Provision of study materials or patients: Y Bai, \\ ZZ Wang, GG Zang, SD Guo, YL Wang; (IV) Collection and assembly of data: Y Bai, YY Jin, Y Liu; (V) Data analysis and interpretation: Y Bai, JM \\ Rivera-Caravaca; (VI) Manuscript writing: All authors; (VII) Final approval of manuscript: All authors. \\ Correspondence to: Ying Bai. Cardiovascular Center, Beijing Tongren Hospital, Capital Medical University, No. 1, Dong Jiao Min Xiang Street, \\ Dongcheng District, Beijing 100730, China. Email: byfeixue@sina.com; Yue Liu, PhD. Department of Cardiology, The First Affiliated Hospital of \\ Harbin Medical University, Harbin, China. Email: liuyue198002@163.com.
}

Background Several scores were available for predicting atrial fibrillation (AF) recurrence post radiofrequency ablation. However, the role of different scores predicting AF recurrence after ablation in patients with concurrent $\mathrm{AF}$ and pulmonary diseases (PDs) remained obscure. Herein, we aimed to investigate their predicting values and differences in patients with concurrent AF and PDs.

Methods: From January 2008 to April 2015, 304 patients with concurrent AF and PDs treated with catheter ablation were divided into 2 groups according to whether they experienced AF recurrence in our centers. Factors related with AF recurrence were explored using Cox regression and scores predicting recurrent $\mathrm{AF}$ were compared in these patients using ROC curves.

Results: During a median of 6-month of follow-up, factors correlating with late AF recurrence included heart failure (HF) history [hazard ratio (HR): 2.79; 95\% confidence interval (CI): 1.49-5.22, P=0.001], current smoking $(1.73 ; 1.13-2.68, \mathrm{P}=0.01)$ and early AF recurrence $(3.85 ; 95 \% \mathrm{CI}: 2.62-5.66, \mathrm{P}<0.001)$ according to univariate Cox regression analysis. When analyzed using multivariate Cox model, HF history (2.21; 1.12-4.37, $\mathrm{P}=0.02)$, hypertension history $(1.54 ; 1.02-2.33, \mathrm{P}=0.04)$ and early AF recurrence (3.90; 2.60-5.85, $\mathrm{P}<0.001)$ were related to late AF recurrence. The BASE-AF2 score had higher c-index than the MB-LATER, APPLE, CHADS2, CHA2DS2-VASc, CAAP-AF and HATCH scores when compared using ROC curves analysis (all $\mathrm{P}<0.05$ ). The optimal point for predicting AF recurrence of the BASE-AF2 score in the ROC analysis was 1 point with sensitivity of $69.03 \%$ and specificity of $60.21 \%$.

Conclusions: The predicting AF recurrence value of BASE-AF2 score was superior to MB-LATER, APPLE, CHADS2, CHA2DS2-VASc, CAAP-AF and HATCH scores in patients with concurrent AF and PDs, which can be an effective and helpful score for making AF treatment decisions.

Keywords: Pulmonary diseases (PDs); catheter ablation; atrial fibrillation recurrence; the BASE-AF2 score

Submitted Feb 23, 2021. Accepted for publication Mar 10, 2021.

doi: $10.21037 /$ apm-21-437

View this article at: http://dx.doi.org/10.21037/apm-21-437 


\section{Introduction}

Pulmonary diseases (PDs) are sometimes concurrent with atrial fibrillation (AF) (1) and trend to increase the AF recurrence rate after catheter ablation $(2,3)$. Therefore, it would be very helpful for making treatment choices in patients with concurrent $\mathrm{AF}$ and PDs by developing scores predicting AF recurrence after catheter ablation. Several scores including BASE-AF2 score (4), HATCH score (5), APPLE score (6), CHADS2 score (7), CHA2DS2-VASc score (7), MB-LATER score (8) and CAAP-AF score (9) were developed for predicting $\mathrm{AF}$ recurrence in recent years, based on different catheter ablation protocols (i.e., cryoablation, circumferential pulmonary vein isolation, etc.). An accumulating body of evidence indicates that PDs are independently associated with AF. Concomitant $\mathrm{PDs}$ in $\mathrm{AF}$ patients are associated with $\mathrm{AF}$ recurrence, ablation outcomes and mortality. Although COPD was a studied variable in HATCH scoring system, none of these scoring systems were specifically evaluated in patients with concurrent $\mathrm{AF}$ and $\mathrm{PDs}$ for $\mathrm{AF}$ recurrence. Among these predicting scores, physicians often feel confused about which to choose for predicting AF recurrence after catheter ablation in patients with concurrent $\mathrm{AF}$ and PDs. Therefore, we designed this study to explore their predicting values and differences in AF recurrence of patients with concurrent AF and PDs when they were treated with catheter ablation.

We present the following article in accordance with the TRIPOD reporting checklist (available at http://dx.doi. org/10.21037/apm-21-437).

\section{Methods}

Three hundred and fifty-eight AF patients with PDs receiving their first radiofrequency ablation between January 2008 and April 2015 were retrospectively reviewed in our centers. Inclusion Criteria is as follows: (I) receiving radiofrequency ablation as described previously $(10,11)$. Briefly speaking, the ' $2 \mathrm{C} 3 \mathrm{~L}$ ' technique is performed with radiofrequency ablation method, which is a fixed ablation approach consisting of bilateral circumferential pulmonary vein antrum isolation (PVAI) and three linear ablation lesion sets across the mitral isthmus, left atrial roof, and cavo-tricuspid isthmus. The procedure was terminated by cardioversion unless a mappable organized atrial tachyarrhythmia was subject to further ablation until it is non-inducible with burst pacing at $200 \mathrm{~ms}$. (II) With
PDs diagnosed according to information provided by the patients, their medical records, and examinations (i.e., chest X-ray photography, pulmonary function test, lung computed tomography, etc.). (III) With age more than 18 years old. AF patients were excluded from this study if they were complicated with acute myocardial infarction or untreated thyroid dysfunction. The study was conducted in accordance with the Declaration of Helsinki (as revised in 2013). Approval was obtained from the ethics committee of Beijing Tongren Hospital, Capital Medical University (No.: TRECKY2020-097) and individual consent for this retrospective analysis was waived.

After excluding 54 patients (15.08\%) without follow-up information, $304 \mathrm{AF}$ patients complicated with PDs were analyzed in the final study, including 189 patients with COPD, 63 with pulmonary tuberculosis, 5 with recurrent pulmonary embolism, 23 with interstitial lung fibrosis, and 24 with other PDs (e.g., 14 lung cancer after lung resection, 7 bronchiectasis, and 3 asbestosis). These patients were divided into $\mathrm{AF}$ recurrence group and no $\mathrm{AF}$ recurrence group.

Seven scores predicting AF recurrence were analyzed in the current study. The exact definition of the BASE-AF2 score (4), the HATCH score (5), the APPLE score (6), the CHADS2 score (7), the CHA2DS2-VASc score (7), the MB-LATER score (8) and the CAAP-AF score (9) were listed in Table $\mathrm{S} 1$ and as follows.

The BASE-AF2 score (4): body mass index (BMI) $>28 \mathrm{~kg} / \mathrm{m}^{2}$ ( 1 point), atrial dilatation $>40 \mathrm{~mm}$ (1 point), current smoking (1 point), early AF recurrence (1 point), duration of $\mathrm{AF}>6$ years ( 1 point), and non-paroxysmal $\mathrm{AF}$ type (1 point).

The HATCH score (5): COPD (1 point), hypertension (1 point), age $\geq 75$ years ( 1 point), heart failure (HF) ( 2 points), and previous transient ischemic attack (TIA) or stroke (2 points).

The APPLE score (6): age $>65$ years ( 1 point), persistent AF (1 point), impaired eGFR $\left(<60 \mathrm{~mL} / \mathrm{min} / 1.73 \mathrm{~m}^{2}\right)$ (1 point), LA diameter $\geq 43 \mathrm{~mm}$ ( 1 point), and $\mathrm{EF}<50 \%$ (1 point).

The CHADS2 score (7): congestive HF (1 point), hypertension ( 1 point), age $>65$ years ( 1 point), diabetes mellitus (1 point), and previous TIA or stroke (2 points).

The CHA2DS2-VASc score (7): congestive HF (1 point), hypertension (1 point), age: $65-74$ years (1 point), diabetes mellitus ( 1 point), previous TIA or stroke ( 2 points), peripheral artery disease ( 1 point), age: $>75$ years ( 2 points), female (1 point). 
The MB-LATER score (8): male gender (1 point), bundle branch block (1 point), left atrium $\geq 47 \mathrm{~mm}$ (1 point), type of $\mathrm{AF}$ (paroxysmal $\mathrm{AF}=0$ point, persistent $\mathrm{AF}=1$ point, and long-standing persistent $\mathrm{AF}=2$ points), early $\mathrm{AF}$ recurrence (1 point).

The CAAP-AF score (9): coronary heart disease (CHD) ( 1 point); LA diameter ( $0-4$ point); age $(0-3$ point); persistent or long-standing AF (1 point); number of antiarrhythmic drugs (AAD) failed ( $0-2$ point); female (1 point).

Follow-up was performed starting from the day on which the patients received RFA until five years after catheter ablation or the end of the follow-up (December, 2015). The patients' ECGs or Holters were collected from inpatient records and outpatient records. Any recording of AF lasting $>30 \mathrm{~s}$ by ECG or Holter was considered as AF recurrence, dividing into early $\mathrm{AF}$ recurrence if less than 3 months and late $\mathrm{AF}$ recurrence if more than 3 months.

\section{Statistical analysis}

Normality assessment of continuous variables was performed using one-sample Kolmogorov-Smirnov test. Continuous variables with normal distribution were indicated as mean \pm standard deviation (SD) and compared using the student's $t$-test. If not, they were reported as medians with interquartile [median $\left(25^{\text {th }}\right.$ percentile $-75^{\text {th }}$ percentile)] and compared with Wilcoxon rank sum test subsequently. Categorical variables were indicated as numbers (percentages) and then comparison was performed by $\chi^{2}$ test. Possible factors related to late recurrent $\mathrm{AF}$ in PDs patients were assessed using Cox regression models. Factors in the multivariate Cox regression model involved age, type of AF, gender, stroke or TIA history, COPD, hypertension (HTN) history, BMI, current smoking, AF duration period, CHD, failed antiarrhythmic drug number, left atrial diameters, HF history, and early AF recurrence. Kaplan-Meier analysis was used to compare $\mathrm{AF}$ free rate between COPD and non-COPD using a log-rank test. Receiver Operator Curve (ROC) Analysis was used to explore the differences in predicting late $\mathrm{AF}$ recurrence between these prediction scores [BASE-AF2 (4), MB-LATER (8), APPLE (6), CHADS2 (7), CHA2DS2VASc (7), CAAP-AF (9), and HATCH scores (5)], with c-index as indicator of predictive values. Overall, a twotailed $\mathrm{P}$ value $<0.05$ was considered to be statistically significant. SPSS software, version 24.0 (IBM Corporation, Armonk, NY, USA), STATA 12.0 (Stata Inc., USA) and
MedCalc software version 18.2.1 were used for the analyses.

\section{Results}

\section{Baseline characteristics}

Three hundred and four AF patients (age: $62.86 \pm 10.55$ years and $68.1 \%$ male) with PDs were included in this study, with $69.7 \%$ paroxysmal AF, $11.2 \%$ previous stroke or TIA, 53.0\% HTN, 4.3\% HF history and 14.1\% coronary artery disease history. Of all the included patients, those in AF recurrence group are more likely to have HF history (Table 1). While baseline variables are balanced between the two groups (COPD vs. non-COPD, all $\mathrm{P}>0.05$ ) (Table S2).

\section{Factors relating to late AF recurrence}

After a median of 6 months (interquartile range: 618 months) follow-up, $113 \mathrm{AF}$ patients experienced AF recurrence, accounting for $37.2 \%$ of the whole population. Factors relating to late AF recurrence included HF history (HR: 2.79; 95\% CI: 1.49-5.22, $\mathrm{P}=0.001$ ), current smoking $(1.73 ; 1.13-2.68, \mathrm{P}=0.01)$ and early $\mathrm{AF}$ recurrence (3.85; 95\% CI: $2.62-5.66, \mathrm{P}<0.001)$ when analyzed using univariate Cox model (Table 2). When analyzed using multivariate Cox model, HF history $(2.21 ; 1.12-4.37$, $\mathrm{P}=0.02)$, HTN history $(1.54 ; 1.02-2.33, \mathrm{P}=0.04)$ and early $\mathrm{AF}$ recurrence $(3.90 ; 2.60-5.85, \mathrm{P}<0.001)$ were related to late $\mathrm{AF}$ recurrence (Table $\mathrm{S} 3$ ).

\section{Scores predicting AF recurrence following catheter ablation}

The BASE-AF2 score had a significantly higher c-index than the MB-LATER, APPLE, CHADS2, CHA2DS2VASc, CAAP-AF, and HATCH scores (all $\mathrm{P}<0.05$ ). The optimal point for predicting AF recurrence of the BASE$\mathrm{AF} 2$ score in the ROC analysis was 1 point with sensitivity of $69.03 \%$ and specificity of $60.21 \%$. The MB-LATER score had a relative higher c-index than the APPLE score and CAAP-AF scores for prediction of AF recurrence. There was no significant difference among the other scores (Figure 1).

\section{Kaplan-Meier analysis}

Included AF patients complicated with COPD showed similar AF recurrence hazard ratio when compared to those with non-COPD (log-rank: $\mathrm{P}=0.71$ ) (Figure 2). 
Table 1 Baseline characteristics

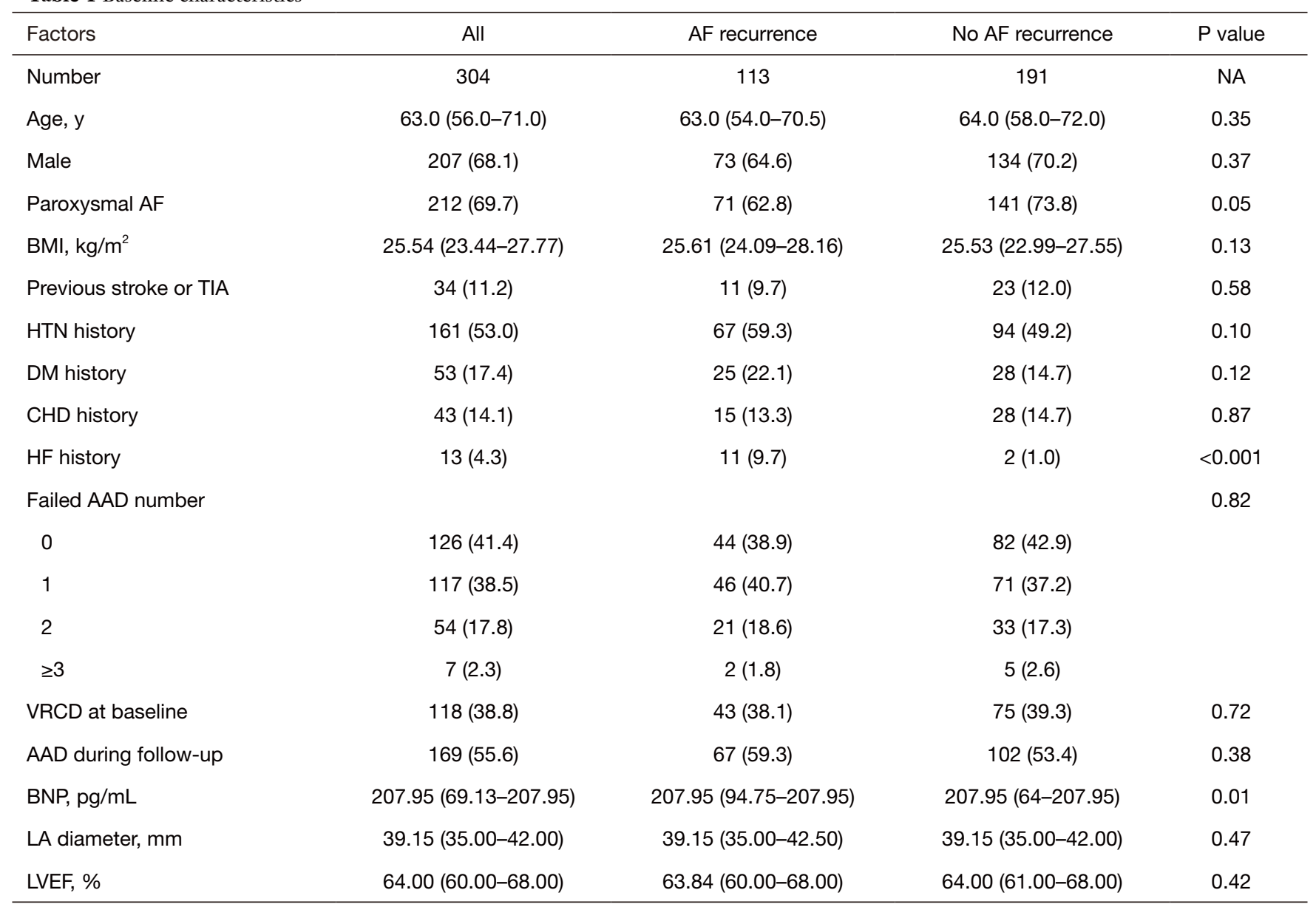

Data were shown as median (interquartile range) or number (\%); BMI, body mass index; COPD, chronic obstructive pulmonary diseases; AF, atrial fibrillation; TIA, transient ischemic attack; BMI, body mass index; HTN, hypertension; DM, diabetes mellitus; CHD, coronary heart diseases; HF, heart failure; AAD, anti-arrhythmic drugs; BNP, type B natriuretic peptide; LA, left atrium; LVEF, left ventricular ejection fraction; VRCD, ventricular rate control drugs.

\section{Discussion}

In this study, the important finding is that HF history, current smoking and early recurrent $\mathrm{AF}$ among clinical factors involved in the prediction scores were found to be significantly related to late recurrent $\mathrm{AF}$ following catheter ablation when analyzed in univariate Cox regression model. The further analysis showed that the predicting ability of BASE-AF2 score was superior to the MB-LATER, APPLE, CHADS2, CHA2DS2-VASc, CAAP-AF and HATCH scores when comparisons were made in patients with concurrent $\mathrm{AF}$ and PDs.

To our knowledge, this is the first study validating these predicting scores in AF patients with PDs, a large group of patients in many centers may not consider for ablation.
Nonetheless, some controversies were shown compared with previous studies. The APPLE score was shown to have a relatively high prediction value in the general population $(6,8)$. Though HF history was shown significantly related to AF recurrence, $\mathrm{LVEF}$ was not related to late AF recurrence in patients with PDs. HF with preserved LVEF was supposed as possible explanations for this controversial phenomenon. Therefore, the APPLE score including LVEF, not congestive HF had relatively lower predicting value compared with the HATCH, CHADS2 and CHA2DS2-VASc scores which take HF as risk factors, though no statistically significant differences among them in this study. Actually, these patients with both AF and PDs were associated with worse outcomes after AF ablation 
Table 2 Cox Regression Analysis of clinical factors predicting AF recurrence

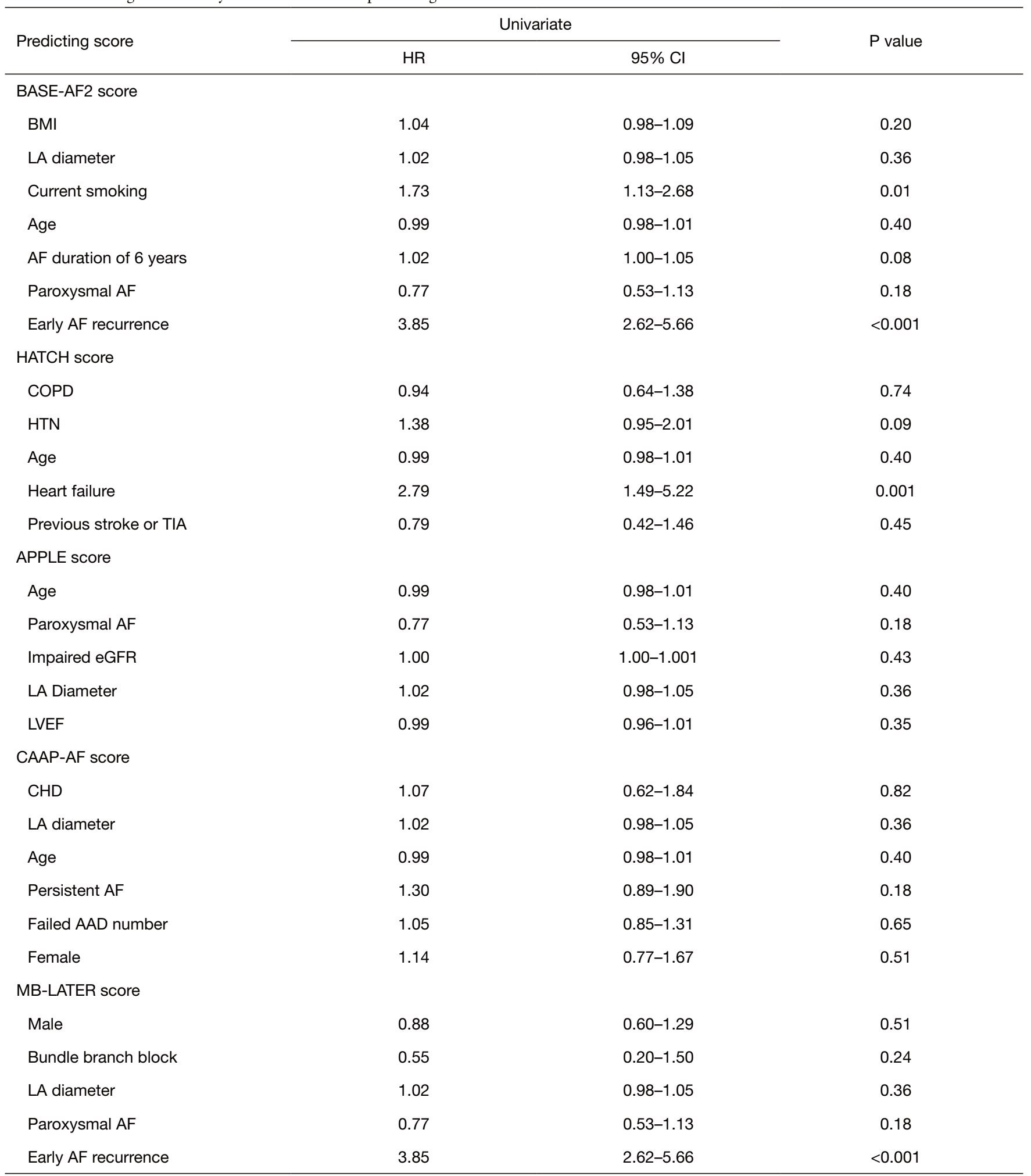

Table 2 (continued) 
Table 2 (continued)

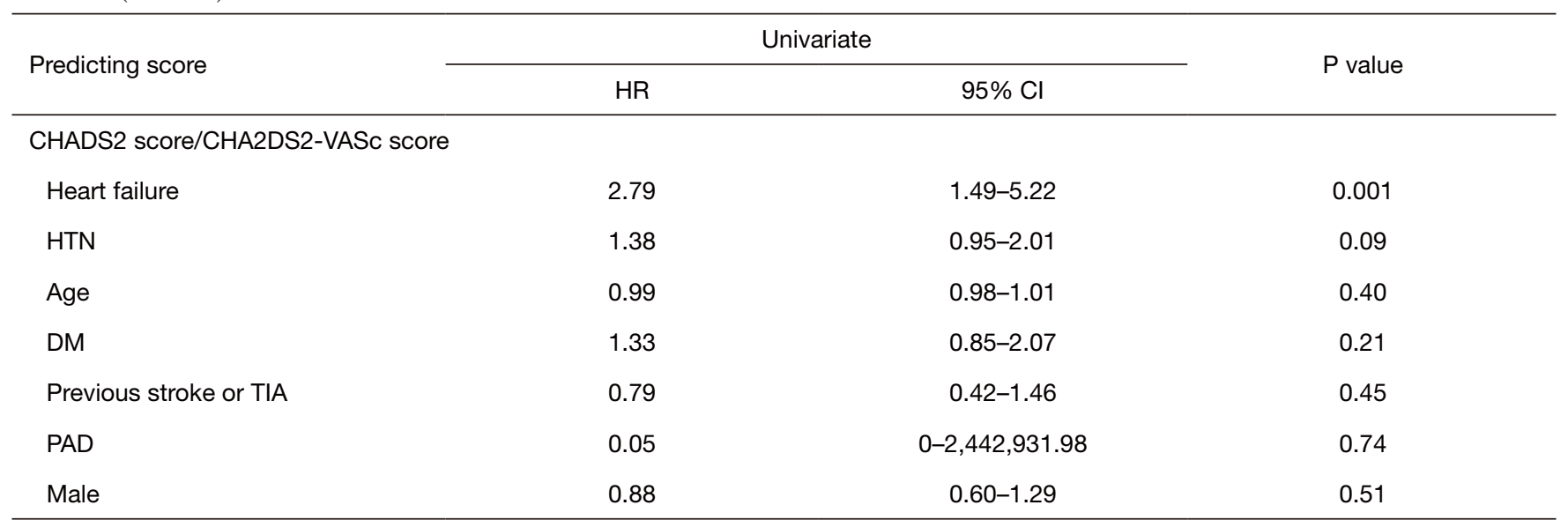

AF, atrial fibrillation; TIA, transient ischemic attack; COPD, chronic obstructive pulmonary diseases; HTN, hypertension; DM, diabetes mellitus; TIA, transient ischemic attack; BMI, body mass index; AAD, anti-arrhythmic drugs; LA, left atrium; eGFR, estimated glomerular filtration rate; LVEF, left ventricular ejection fraction; CHD, coronary heart disease.

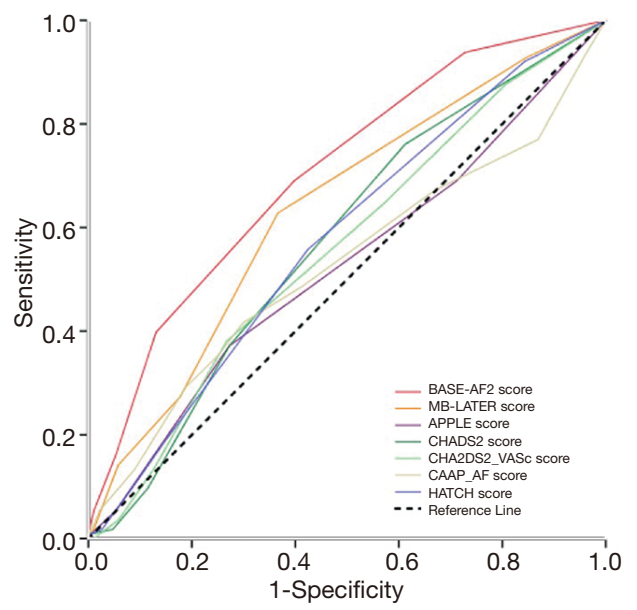

\begin{tabular}{|c|c|c|c|c|c|c|c|}
\hline Predicting scores & c-index $(95 \% \mathrm{Cl})$ & & & & & & \\
\hline BASE-AF2 & $0.70(0.65-0.76)$ & BASE-AF2 & & & & & \\
\hline MB-LATER & $0.64(0.58-0.69)$ & 0.01 & MB-LATER & & & & \\
\hline APPLE & $0.53(0.47-0.58)$ & $<0.0001$ & 0.0004 & APPLE & & & \\
\hline CHADS2 & $0.58(0.52-0.63)$ & 0.002 & 0.16 & 0.22 & CHADS2 & & \\
\hline CHA2DS2-VASc & $0.56(0.50-0.62)$ & 0.001 & 0.12 & 0.37 & 0.42 & CHA2DS2-VASC & \\
\hline CAAP-AF & $0.53(0.47-0.59)$ & $<0.0001$ & 0.0006 & 0.93 & 0.23 & 0.4 & CAAP-AF \\
\hline HATCH & $0.58(0.52-0.63)$ & 0.001 & 0.16 & 0.21 & 0.97 & 0.53 & 0.22 \\
\hline
\end{tabular}

Figure 1 Receiver operator curves on the risk of AF recurrence among BASE_AF2, MB_LATER, APPLE, CHADS2, CHA2DS2-VASc, CAAP-AF and HATCH scores. BASE-AF2: B, Body body mass index $>28 \mathrm{~kg} / \mathrm{m}^{2}$; A, atrial dilatation $>40 \mathrm{~mm}$; S, current smoking; E, early AF recurrence; A, duration of AF 6 years; F, non-paroxysmal AF type; MB-LATER: M, male gender; B, bundle branch block; LA, left atrial diameter $\geq 47 \mathrm{~mm}$; T, type of AF (paroxysmal, persistent, and long-standing persistent), ER, early AF recurrence; APPLE: A, age $>65$ years; $\mathrm{P}$, persistent AF; P, impaired eGFR $<60 \mathrm{~mL} / \mathrm{min} / 1.73 \mathrm{~m}^{2}$; L, left atrial diameter $\geq 43 \mathrm{~mm}$; E, EF <50\%; CHADS2: C, congestive heart failure; H, hypertension; A, age $>65$ years; D, diabetes mellitus ; S, previous TIA or stroke; CHA2DS2-VASc: C, congestive heart failure, H, hypertension; A, age: 65-74 years; D, diabetes mellitus; S, previous TIA or stroke; V, peripheral artery disease; A, age: >75 years; S, female sex; CAAP-AF: C, coronary heart disease; A, left atrial diameter; A, age; P, persistent or long-standing AF; A, number of antiarrhythmic drugs (AAD) failed; F, female sex; HATCH: H, heart failure; A, age $\geq 75$ years; T, previous transient ischemic attack (TIA) or stroke; C, COPD; H, hypertension. 


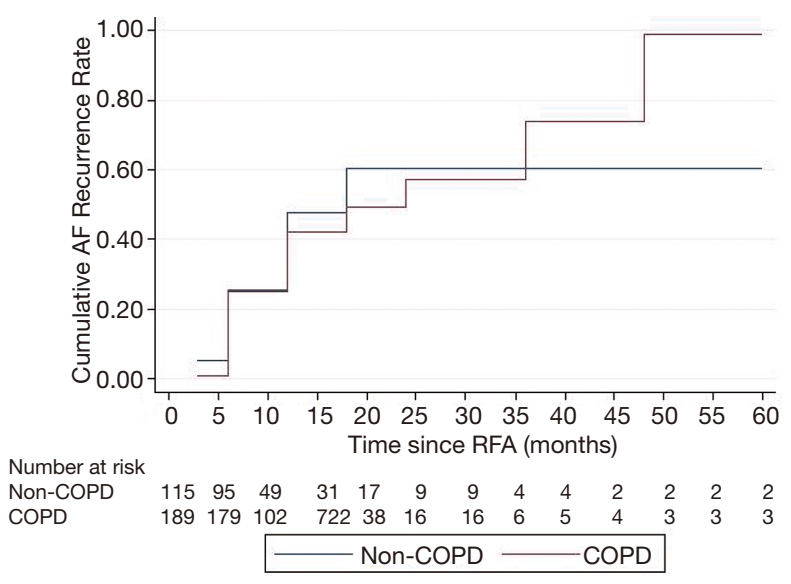

Figure 2 Patients with COPD (red line) had similar atrial fibrillation free rate compared with other kind of CLD patients (non-COPD, blue line) using Kaplan-Meier analysis (log-rank: $\mathrm{P}=0.71$ ). COPD, chronic obstructive pulmonary disease; CLD, chronic lung disease.

because PDs could be the underlying disease leading to $\mathrm{AF}$ (1). The PDs was not resolved even if the AF ablation was performed, which made AF hardly treated. Another possible reason to explain the worse performance in these patients was that AF patients with PDs had somewhat different characteristics. As LA was one important factor involved in most of the scores (12), but right atrial diameter was neglected which was more often affected by the PDs, which contribute a lot to the recurrent $\mathrm{AF}$ after ablation (13).

The BASE-AF2 score was generated in AF patients with cryoablation treatment for predicting AF recurrence using a Turkish cohort (4), but not validated in those with PDs treated with catheter ablation yet. In the present study, we confirmed that the BASE-AF2 score was also applicable to patients with concurrent $\mathrm{AF}$ and $\mathrm{PDs}$ for predicting $\mathrm{AF}$ recurrence following catheter ablation, and had a higher prediction effect compared with the other scores, including the MB-LATER score which showed superior predicting ability of very late recurrent AF (longer than 12 months) (8), even if both of these scores involved early AF recurrence, which contributed greatly to late AF recurrence (14). One possible reason was suspected that smoking involved in the BASE-AF2 score was also risk factor contributing to incidence of PDs (15). Though patients with concurrent $\mathrm{AF}$ and PDs are clinically common, they are not usually treated with catheter ablation. Catheter ablation may be an appropriate choice for those with high possibility of restoring sinus rhythm (e.g., high BASE-AF2 score), after integrated consideration of the score and the clinical evaluation. Unfortunately, though the BASE-AF2 and MB-LATER scores were superior to other risk scores for predicting post-ablation AF recurrence, both the scores cannot be calculated before ablation due to involvement of early AF recurrence (in the first three months after ablation) as part of the score. Therefore, antiarrhythmic drugs were advised to be used once the patients had early $\mathrm{AF}$ recurrence in order to reduce late $\mathrm{AF}$ recurrence risk. There is room for improvement in the progress of these scores predicting recurrent $\mathrm{AF}$ in the subgroup of patients with $\mathrm{AF}$ and concomitant $\mathrm{PDs}$.

Initially, the HATCH score was explored for predicting risk of paroxysmal AF progressing to persistent $\mathrm{AF}$. COPD was one kind of PDs, which was independently involved in the HATCH score. However, the HATCH score was shown to have a relatively low c-index in predicting $\mathrm{AF}$ recurrence in the current cohort. Moreover, whether COPD had a different effect compared with other kinds of PDs (nonCOPD) on the outcome of RFA was unclear. In the current study, no impact of COPD on the outcome of RFA was seen using univariate Cox regression analysis and the result was further confirmed by comparing $\mathrm{AF}$ free rate between COPD and non-COPD using Kaplan-Meier analysis.

There are several limitations to be mentioned for this study. First, COPD patients constitute $62.17 \%$ of the study group. Thus, the results of the study are largely driven by the COPD patients which limits generalization of the study findings. However, COPD patients accounting for the majority of the study has minimized the possible heterogeneity caused by PDs contributing to outcomes post ablation. Second, the PDs status were hardly accounted for as no uniform indicator was available for each kind of PDs, though the incorporation of PDs stratification stage may possibly provide added value, which is hardly obtained for the current analysis. Third, many episodes known as asymptomatic $\mathrm{AF}$ could be neglected by the patients and hardly recorded, which raises significant concern about the validity of the findings, though ECGs and Holter were performed whenever possible during each follow-up. Fourth, patient-reported PDs history were also included in the study, however, examinations such as chest $\mathrm{X}$-rays was used for adjudication during in-hospital stay, which increased the robustness of the diagnosis. Moreover, the studied PDs cohort is very selective and not representative of a wider AF population. However, this study proved that the BASE-AF2 score was useful in predicting AF recurrence following ablation in a specific PDs cohort with histories of 
mild to severe PDs. Fifth, patients with acute myocardial infarction were excluded and baseline CHD was balanced to minimize the influence caused by cardiovascular risk before ablation, as the 'success' of the procedure is likely to be significantly affected. Sixth, even if the topic was very useful and important, it is not easy to validate the scores in the patients with concurrent $\mathrm{AF}$ and $\mathrm{PDs}$, as these patients are not usually treated with catheter ablation, which might lead to the source of bias and limit the generalizability of the findings. Therefore, even if the analysis was based on a solid analytic process, a larger confirmation of their results is still needed due to the relatively small number of patients presented in the current study. Seventh, as known by everyone, all the scores are shown overall modest predictive performances as risk scores have a limited power in predicting clinical events in AF patients. Indeed, it is necessary to integrate the scores in the current clinical evaluation of patients, but not substitute the physicians' clinical decision process. Eighth, several scores were not validated in the current analysis because such data were available only for part of the patients, such as left atrial size in the ALARMEc score and left atrial volume in the ATLAS score.

\section{Conclusions}

Our results showed that HF history, current smoking and early recurrent $\mathrm{AF}$ among clinical factors involved in the prediction scores is related with late recurrent $\mathrm{AF}$ post catheter ablation. Moreover, The BASE-AF2 score had higher predicting values than the MB-LATER, APPLE, CHADS2, CHA2DS2-VASc, CAAP-AF and HATCH scores in identifying recurrent $\mathrm{AF}$ after ablation when used in patients with concurrent $\mathrm{AF}$ and PDs, which can be an effective and helpful score for making AF treatment decisions.

\section{Acknowledgments}

Funding: This study was supported by grants from National Natural Science Foundation of China (81800291, 81501486) and Beijing Hospitals Authority Youth Programme (QML20180207).

\section{Footnote}

Reporting Checklist: The authors have completed the TRIPOD reporting checklist. Available at http://dx.doi. org/10.21037/apm-21-437

Data Sharing Statement: Available at http://dx.doi. org/10.21037/apm-21-437

Peer Review File: Available at http://dx.doi.org/10.21037/ apm-21-437

Conflicts of Interest: All authors have completed the ICMJE uniform disclosure form (available at http://dx.doi. org/10.21037/apm-21-437). Dr. José Miguel RiveraCaravaca has received a grant from Sociedad Española de Trombosis y Hemostasia (grant for short international training stays 2020) and the First Contact Initiative Grant 2020 from the European Society of Cardiology Council on Basic Cardiovascular Science. The other authors have no conflicts of interest to declare.

Ethical Statement: The authors are accountable for all aspects of the work in ensuring that questions related to the accuracy or integrity of any part of the work are appropriately investigated and resolved. The study was conducted in accordance with the Declaration of Helsinki (as revised in 2013). Approval was obtained from the ethics committee of Beijing Tongren Hospital, Capital Medical University (No.: TRECKY2020-097) and individual consent for this retrospective analysis was waived.

Open Access Statement: This is an Open Access article distributed in accordance with the Creative Commons Attribution-NonCommercial-NoDerivs 4.0 International License (CC BY-NC-ND 4.0), which permits the noncommercial replication and distribution of the article with the strict proviso that no changes or edits are made and the original work is properly cited (including links to both the formal publication through the relevant DOI and the license). See: https://creativecommons.org/licenses/by-nc-nd/4.0/.

\section{References}

1. Shih HT, Webb CR, Conway WA, et al. Frequency and significance of cardiac arrhythmias in chronic obstructive lung disease. Chest 1988;94:44-8.

2. Gu J, Liu X, Tan H, et al. Impact of chronic obstructive pulmonary disease on procedural outcomes and quality of life in patients with atrial fibrillation undergoing catheter ablation. J Cardiovasc Electrophysiol 2013;24:148-54.

3. Kanmanthareddy A, Vallakati A, Reddy Yeruva M, 
et al. Pulmonary vein isolation for atrial fibrillation in the postpneumonectomy population: a feasibility, safety, and outcomes study. J Cardiovasc Electrophysiol 2015;26:385-9.

4. Canpolat U, Aytemir K, Yorgun H, et al. A proposal for a new scoring system in the prediction of catheter ablation outcomes: promising results from the Turkish Cryoablation Registry. Int J Cardiol 2013;169:201-6.

5. de Vos CB, Pisters R, Nieuwlaat R, et al. Progression from paroxysmal to persistent atrial fibrillation clinical correlates and prognosis. J Am Coll Cardiol 2010;55:725-31.

6. Kornej J, Hindricks G, Shoemaker MB, et al. The APPLE score: a novel and simple score for the prediction of rhythm outcomes after catheter ablation of atrial fibrillation. Clin Res Cardiol 2015;104:871-6.

7. Letsas KP, Efremidis M, Giannopoulos G, et al. CHADS2 and CHA2DS2-VASc scores as predictors of left atrial ablation outcomes for paroxysmal atrial fibrillation. Europace 2014;16:202-7.

8. Mujovic N, Marinkovic M, Markovic N, et al. Prediction of very late arrhythmia recurrence after radiofrequency catheter ablation of atrial fibrillation: The MB-LATER clinical score. Sci Rep 2017;7:40828.

9. Winkle RA, Jarman JW, Mead RH, et al. Predicting atrial fibrillation ablation outcome: The CAAP-AF score. Heart

Cite this article as: Bai Y, Wang ZZ, Zhang GG, Guo SD, Rivera-Caravaca JM, Wang YL, Jin YY, Liu Y. Validating scores predicting atrial fibrillation recurrence post catheter ablation in patients with concurrent atrial fibrillation and pulmonary diseases. Ann Palliat Med 2021;10(4):4299-4307. doi: 10.21037/ apm-21-437
Rhythm 2016;13:2119-25.

10. Dong JZ, Sang CH, Yu RH, et al. Prospective randomized comparison between a fixed '2C3L' approach vs. stepwise approach for catheter ablation of persistent atrial fibrillation. Europace 2015;17:1798-806.

11. Tang RB, Liu DL, Dong JZ, et al. High-normal thyroid function and risk of recurrence of atrial fibrillation after catheter ablation. Circ J 2010;74:1316-21.

12. Shin SH, Park MY, Oh WJ, et al. Left atrial volume is a predictor of atrial fibrillation recurrence after catheter ablation. J Am Soc Echocardiogr 2008;21:697-702.

13. Bai Y, Zhao Y, Li J, et al. Association of peak atrial longitudinal strain with atrial fibrillation recurrence in patients with chronic lung diseases following radiofrequency ablation. Intern Med J 2018;48:851-9.

14. Andrade JG, Khairy P, Macle L, et al. Incidence and significance of early recurrences of atrial fibrillation after cryoballoon ablation: insights from the multicenter Sustained Treatment of Paroxysmal Atrial Fibrillation (STOP AF) Trial. Circ Arrhythm Electrophysiol 2014;7:69-75.

15. Salvi S. Tobacco smoking and environmental risk factors for chronic obstructive pulmonary disease. Clin Chest Med 2014;35:17-27. 
Supplementary

Table S1 Factors included in the scores predicting AF recurrence after ablation

\begin{tabular}{|c|c|c|c|c|c|c|c|}
\hline Risk scores & $\mathrm{HATCH}$ & BASE-AF2 & CHADS2 & CHA2DS2-VASc & APPLE & MB-LATER & CAAP-AF \\
\hline Age & + & & + & + & + & & + \\
\hline Sex & & & & + & & + & + \\
\hline AF type & & + & & & + & + & + \\
\hline AF history & & + & & & & & \\
\hline Left atrial diameter & & + & & & + & + & + \\
\hline Early recurrence & & + & & & & + & \\
\hline Hypertension & + & & + & + & & & \\
\hline $\mathrm{CHD}$ & & & & & & & + \\
\hline Heart failure & + & & + & + & + & & \\
\hline Diabetes Mellitus & & & + & + & & & \\
\hline Chronic kidney disease & & & & & + & & \\
\hline Smoking & & + & & & & & \\
\hline Stroke/TIA & + & & + & + & & & \\
\hline Body mass index & & + & & & & & \\
\hline COPD & + & & & & & & \\
\hline Failed AAD & & & & & & & + \\
\hline Vascular disease & & & & + & & & \\
\hline Bundle branch block & & & & & & + & \\
\hline
\end{tabular}

$\mathrm{AF}$, atrial fibrillation; CHD, coronary heart disease; TIA, transient ischemia attack; COPD, chronic obstructive pulmonary disease; AAD, anti-arrhythmic drugs. 
Table S2 Baseline Characteristics

\begin{tabular}{|c|c|c|c|c|}
\hline & All & COPD & Non-COPD & $P$ value \\
\hline Number & 304 & 189 & 115 & \\
\hline Age & & & & 0.94 \\
\hline Median & 63.0 & 64.00 & 62.00 & \\
\hline IQR & $56.0-71.0$ & $57.00-70.50$ & $54.00-72.00$ & \\
\hline Sex & & & & 0.10 \\
\hline Female & 97 & 67 & 30 & \\
\hline Male & 207 & 122 & 85 & \\
\hline AF type & & & & 0.09 \\
\hline Paroxysmal AF & 212 & 125 & 87 & \\
\hline Persistent AF & 92 & 64 & 28 & \\
\hline BMI & & & & 0.18 \\
\hline Median & 25.54 & 25.63 & 25.54 & \\
\hline IQR & $23.44-27.77$ & $23.49-28.29$ & $23.25-27.12$ & \\
\hline Previous stroke or TIA & & & & 1.00 \\
\hline Yes & 34 & 21 & 13 & \\
\hline No & 270 & 168 & 102 & \\
\hline HTN history & & & & 0.41 \\
\hline Yes & 161 & 104 & 57 & \\
\hline No & 143 & 85 & 58 & \\
\hline DM history & & & & 0.12 \\
\hline Yes & 53 & 38 & 15 & \\
\hline No & 251 & 151 & 100 & \\
\hline CHD history & & & & 0.61 \\
\hline Yes & 43 & 25 & 18 & \\
\hline No & 261 & 164 & 97 & \\
\hline HF history & & & & 0.14 \\
\hline Yes & 13 & 11 & 2 & \\
\hline No & 291 & 178 & 113 & \\
\hline BNP & & & & 0.71 \\
\hline median & 207.95 & 207.95 & 207.95 & \\
\hline IQR & 69.13-207.95 & $68.00-207.95$ & $70.50-207.95$ & \\
\hline LA diameter & & & & 0.31 \\
\hline median & 39.15 & 39.15 & 39.00 & \\
\hline IQR & $35.00-42.00$ & $35.50-42.00$ & $34.00-43.00$ & \\
\hline LVEF & & & & 0.41 \\
\hline median & 64.00 & 64.00 & 64.00 & \\
\hline IQR & $60.00-68.00$ & $60.00-68.00$ & $60.00-69.00$ & \\
\hline
\end{tabular}

IQR, interquartile range; COPD, chronic obstructive pulmonary diseases; $A F$, atrial fibrillation; TIA, transient ischemic attack; BMI, body mass index; HTN, hypertension; DM, diabetes mellitus; CHD, coronary heart diseases; HF, heart failure; BNP, type B natriuretic peptide; LA, left atrium; LVEF, left ventricular ejection fraction. 
Table S3 Cox Regression Analysis of clinical factors associated with recurrent AF

\begin{tabular}{|c|c|c|c|c|c|c|}
\hline & \multicolumn{3}{|c|}{ Univariate } & \multicolumn{3}{|c|}{ Multivariate } \\
\hline & $\mathrm{HR}$ & $(95 \% \mathrm{Cl})$ & $P$ value & $\mathrm{HR}$ & $(95 \% \mathrm{Cl})$ & $P$ value \\
\hline Age & 0.99 & $0.98-1.01$ & 0.40 & 0.99 & $0.97-1.01$ & 0.20 \\
\hline Male & 0.88 & $0.60-1.29$ & 0.51 & 0.95 & $0.61-1.48$ & 0.80 \\
\hline Heart failure & 2.79 & $1.49-5.22$ & 0.001 & 2.21 & $1.12-4.37$ & 0.02 \\
\hline Previous stroke or TIA & 0.79 & $0.42-1.46$ & 0.45 & 0.80 & $0.42-1.50$ & 0.48 \\
\hline COPD & 0.94 & $0.64-1.38$ & 0.74 & 0.72 & $0.48-1.08$ & 0.11 \\
\hline HTN & 1.38 & $0.95-2.01$ & 0.09 & 1.54 & $1.02-2.33$ & 0.04 \\
\hline BMI & 1.04 & $0.98-1.09$ & 0.20 & 0.99 & $0.93-1.05$ & 0.66 \\
\hline LA-Diameter & 1.02 & $0.98-1.05$ & 0.36 & 1.00 & $0.96-1.03$ & 0.86 \\
\hline Paroxysmal AF & 0.77 & $0.53-1.13$ & 0.18 & 0.95 & $0.62-1.47$ & 0.82 \\
\hline CHD & 1.07 & $0.62-1.84$ & 0.82 & 1.18 & $0.66-2.31$ & 0.58 \\
\hline Failed AAD number & 1.05 & $0.85-1.31$ & 0.65 & 0.94 & $0.61-1.46$ & 0.79 \\
\hline Current Smoking & 1.73 & $1.13-2.68$ & 0.01 & 1.63 & $0.96-2.78$ & 0.07 \\
\hline Early AF recurrence & 3.85 & $2.62-5.66$ & $<0.001$ & 3.90 & $2.60-5.85$ & $<0.001$ \\
\hline
\end{tabular}

AF, atrial fibrillation; TIA, transient ischemic attack; COPD, chronic obstructive pulmonary diseases; HTN, hypertension; BMI, body mass index; AAD, anti-arrhythmic drug; LA, left atrium. 